\title{
Características y evolución de las fracturas de cadera operadas en el Banco de Prótesis (enero-diciembre 2013)
}

\author{
Dras. Marianela Maiche*, Marcela Hernández† , Beatriz Mendoza ${ }^{\ddagger}$
}

\section{Resumen}

Introducción: la fractura de cadera por fragilidad es considerada un marcador de enfermedad osteoporótica en la población añosa. Determina una elevada morbimortalidad y altos costos en salud.

Objetivos: analizar características y evolución posterior a una fractura de cadera por fragilidad en pacientes $\geq 65$ años asistidos en el Banco de Prótesis durante el período enero-junio 2013.

Método: estudio de seguimiento de cohorte histórica, constituido por 184 pacientes. Los datos se obtuvieron de las historias clínicas y el seguimiento se realizó telefónicamente. El tiempo de seguimiento máximo fue de 84 semanas.

Resultados: sexo femenino ( $82,6 \%$ ), con una mediana de edad de 80,7 años para los hombres y 82,7 para las mujeres. Las comorbilidades más frecuentes fueron hipertensión arterial y enfermedad cardiovascular. No hubo diferencias en la localización derecha o izquierda, la mayoría correspondió a fracturas de cuello femoral. La mediana del tiempo de resolución fue de 9 días para artroplastia y 11 días para osteosíntesis. La caída fue la determinante en $94,5 \%$ de los casos. El $26 \%$ de los pacientes tenían fracturas previas por fragilidad. El 85\% de los individuos desconocía padecer osteoporosis (OP) y el $69 \%$ no recibía tratamiento para esta enfermedad. El $9 \%$ fue institucionalizado, $77 \%$ logró retomar la deambulación y $15 \%$ falleció en el período de seguimiento.

Conclusiones: no se reconoce ni se trata la OP en los pacientes con fractura de cadera por fragilidad.

Palabras clave: Fracturas de cadera

Osteoporosis

Anciano

Artoplastia

Fracturas osteoporóticas

Key words: $\quad$ Hip fractures

Osteoporosis

Aged

Arthroplasty

Osteoporotic fractures

\footnotetext{
* Internista, Ex Asistente Clínica Médica 1. Médica endocrinóloga. Facultad de Medicina. Universidad de la República, Uruguay. † Internista, Ex Residente Medicina Interna. Médica endocrinóloga. Facultad de Medicina. Universidad de la República, Uruguay. ‡ Prof. Agda. Cátedra de Endocrinología y Metabolismo. Facultad de Medicina. Hospital de Clínicas. Universidad de la República, Uruguay. Banco de Prótesis. Clínica de Endocrinología y Metabolismo.

Facultad de Medicina, Universidad de la República. Montevideo, Uruguay.

Correspondencia: Marianela Maiche Marini. Caldas 1706. Casa 10. CP: 11400. Correo electrónico: marianela.maiche@gmail.com

Los autores declaran no poseer conflicto de intereses.

Aprobado por el Comité de Ética del Banco de Protesis

Recibido: 11/3/19

Aprobado: 10/6/19
} 


\section{Introducción}

La fractura de cadera es una de las lesiones óseas más devastadoras en la población añosa debido a su elevada morbimortalidad, carga social y económica que determina.

El conocimiento de la enfermedad osteoporótica como base de esta y otras fracturas por fragilidad es fundamental para implementar políticas sanitarias destinadas a disminuir su incidencia. En Uruguay no hay registros de fractura de cadera ni datos sobre la evolución posterior que incluyan índices de prescripción de tratamiento médico antiosteoporótico. Los únicos datos disponibles provienen del FNR (Fondo Nacional de Recursos), institución creada con carácter de persona pública no estatal, que brinda cobertura financiera a procedimientos de medicina altamente especializada y a medicamentos de alto costo para toda la población que radica en nuestro país. La resolución quirúrgica mediante artroplastia con implante protésico debe ser previamente autorizada por esta institución. Según la base de datos del FNR ${ }^{(1)}$, en el año 2013 se autorizaron 1.032 artroplastias de cadera por fractura. Se desconocen datos de las restantes fracturas no resueltas mediante implante protésico.

El objetivo de este estudio es analizar las características y la evolución posterior a una fractura de cadera por fragilidad, en pacientes mayores de 65 años, intervenidos quirúrgicamente en un centro de medicina altamente especializada (IMAE) en el período enero a junio de 2013.

\section{Marco teórico}

Se define osteoporosis (OP) como una enfermedad esquelética sistémica, caracterizada por baja masa ósea y deterioro de la microarquitectura, con el consiguiente aumento en la fragilidad del hueso y mayor susceptibilidad a sufrir fracturas. El diagnóstico de esta enfermedad puede ser clínico frente a una fractura por fragilidad o por densitometría ósea, o ambos. Para varones mayores de 50 años y mujeres posmenopáusicas, la ISCD (International Society Clinical Densitometry) sugiere el uso de T-score para realizar diagnóstico densitométrico. Se define T-score como el número de desvíos estándar por arriba o por debajo de la media de la DMO (densidad mineral ósea) comparada con población adulta joven, normal, del mismo sexo y raza según NHANES ${ }^{(2)}$. Cuando el valor de T-score es $\geq-2,5$ se considera OP, valores entre -1 y $-2,5$ baja masa ósea y normal cuando con T-score $\geq-1 \mathrm{DE}$.

La Organización Mundial de la Salud (OMS) define la fractura osteoporótica o por fragilidad como aquella causada por un trauma que sería insuficiente para fracturar un hueso normal; por ejemplo, si se trata de una caída desde una altura inferior a la propia estatura del paciente.

La incidencia de la fractura de cadera se encuentra en aumento, consecuencia en parte de la mayor expectativa de vida de la población a nivel mundial. Las caídas y la OP son las causas más importantes en su génesis. La fractura de cadera ocurre en ambos sexos, si bien predomina fundamentalmente en mujeres de raza blanca, posmenopáusicas, y en la octava década de la vida. Es considerada la complicación más temida de la $\mathrm{OP}$, reduce la esperanza de vida en 1,8 años en comparación con la población general, ajustado por edad y sexo ${ }^{(3)}$. De los pacientes que sobreviven al episodio fracturario, solo la mitad vuelve a caminar en forma independiente, $20 \%$ requiere cuidados de larga duración, con alto riesgo de institucionalización y menos de la mitad retoma su capacidad funcional previa a la fractura ${ }^{(4)}$.

Si bien la OP no presenta signos ni síntomas hasta que se produce la primera fractura, una vez que esta ocurre tenemos la oportunidad única de realizar prevención secundaria dado que aproximadamente el $50 \%$ de las personas con una fractura osteoporótica padecerá otra. Este riesgo es máximo en el primer año, perdura por más de diez años y es independiente de la densidad mineral ósea ${ }^{(5)}$.

Pese a la disponibilidad en Uruguay de fármacos seguros y eficaces para la prevención de fracturas, la mayoría de los pacientes con fractura de cadera no reciben tratamiento adecuado para la $\mathrm{OP}^{(6-8)}$. Importantes estu$\operatorname{dios}^{(9,10)}$ han demostrardo el beneficio que ofrece el tratamiento médico en cuanto a la disminución del riesgo de muerte y de nuevas fracturas clínicas. El tratamiento quirúrgico de las fracturas de fémur proximal se realiza mediante artroplastia (sustitución total o parcial de la articulación mediante un implante protésico) u osteosíntesis (reducción y fijación de la fractura mediante implante de distintos dispositivos).

\section{Objetivos del estudio}

Analizar las características y la evolución posterior a una fractura de cadera por fragilidad en pacientes mayores de 65 años intervenidos quirúrgicamente en el Banco de Prótesis en el período enero a junio de 2013.

\section{Pacientes y método}

\section{Diseño del estudio}

Se trata de un estudio descriptivo, longitudinal, de cohorte histórica, realizado en un único centro, el Banco de Prótesis.

Fue evaluado y aprobado por el Comité de Éica de dicha institución. El Banco de Prótesis es un centro quirúrgico en el que funciona el principal IMAE que realiza 


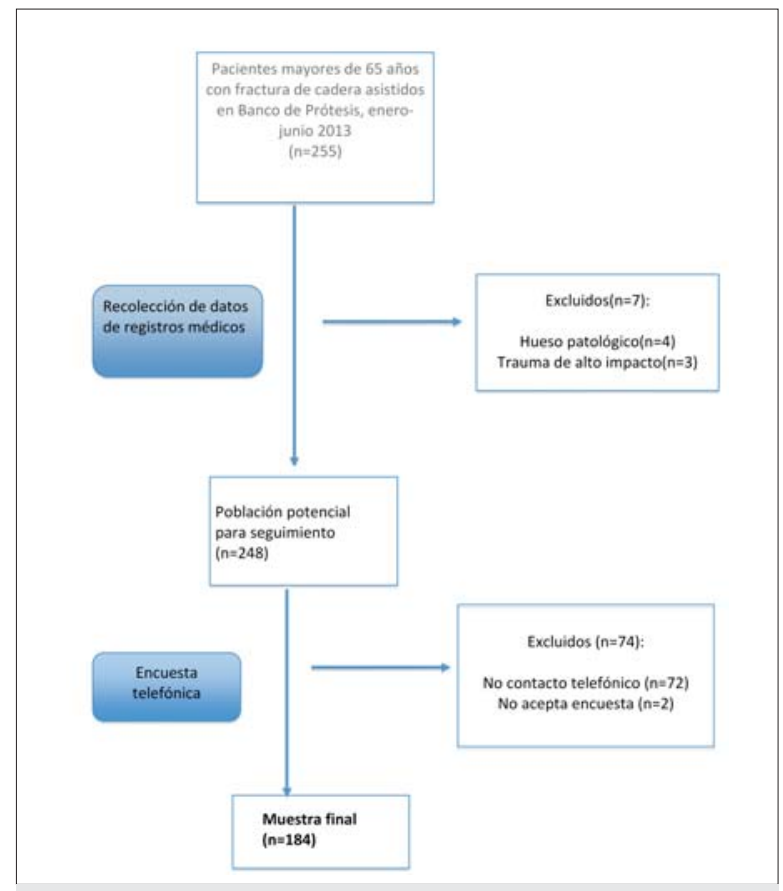

Figura 1. Proceso de selección de la muestra.

las cirugías de sustitución osteoarticular del cuello de fémur en Uruguay.

\section{Pacientes}

Se incluyeron todos los pacientes mayores de 65 años, con fractura de cadera por fragilidad, que ingresaron para resolución quirúrgica al Banco de Prótesis en el período enero a junio de 2013. Se excluyeron pacientes con fractura de cadera de alto impacto, con fractura sobre hueso sustituido por procesos neoplásicos y aquellos en los cuales no se logró realizar contacto telefónico o se negaron a brindar datos.

\section{Procedimiento del estudio}

Se revisaron todas las historias clínicas y radiografías y se contactó telefónicamente al paciente o familiares que vivieran en el domicilio. Se realizó encuesta en base a cuestionario preestablecido, previo consentimiento verbal informado. Se consignaron las siguientes variables: edad, sexo, comorbilidades, localización y topografía de la fractura, tiempo de resolución quirúrgico, caída, conocimiento del diagnóstico de OP, tratamiento antiosteoporótico, fracturas previas y posteriores, evolución del paciente (institucionalización, grado de deambulación y mortalidad). Se definió tiempo de resolución de fractura como el lapso entre el evento y su resolución quirúrgica. Las comorbilidades analizadas fueron las descritas en la historia clínica de la institución. El período de seguimiento fue entre 36 y 84 semanas posterior
Tabla 1. Distribución por edad y sexo. $\mathrm{N}=184$

\begin{tabular}{lcc}
\hline & Frecuencia \% & Edad años \\
\hline Mujeres & 82,61 & 82,70 \\
Hombres & 17,39 & 80,72 \\
\hline
\end{tabular}

al evento fracturario. Se definió la OP del punto de vista clínico como la presencia de fractura ósea por fragilidad.

Se consideró prescripción de tratamiento médico al consumo de suplemento de calcio, vitamina D o bifosfonatos, ya sea en monoterapia o en combinación.

\section{Análisis estadístico}

Para la elaboración estadística se utilizó R Project 3.1.0 en un entorno R-Studio sobre Mac OS X. Para un mejor análisis y discusión, los datos se presentaron en figuras y tablas.

\section{Resultados}

Se enrolaron 255 pacientes en el período de tiempo establecido. Se excluyeron siete pacientes (cuatro por fractura sobre hueso con sustitución maligna y tres por fractura de alto impacto). En 74 pacientes no se pudo realizar encuesta telefónica (72 por no disponer de contacto y dos porque no aceptaron participar en el estudio). La muestra final quedó constituida por 184 pacientes en base a los objetivos del estudio (figura 1).

Hubo predominio del sexo femenino $(82,61 \%)$ sobre el masculino $(17,39 \%)$, con una mediana de edad al momento de la fractura de cadera de 82,3 años, sin diferencias significativas entre ambos $(\mathrm{p}=0,10)($ tabla 1$)$.

Con respecto a las comorbilidades, la más frecuente fue la hipertensión arterial (HTA), seguida de la enfermedad cardiovascular (ECV) y neurológica (tabla 2).

Las asociaciones de comorbilidades más frecuentes fueron: HTA con ECV en 24,46\%, seguida de HTA y patología neurológica (17,39\%), e HTA junto a diabetes millitus (DM) en 17,39\%. La mediana de asociación de comorbilidades fue de 2 .

En cuanto a la localización de la fractura, el 56,67\% correspondió a la cadera izquierda y $43,33 \%$ a fractura de cadera derecha, sin diferencia significativa $(\mathrm{p}=0,07)$. Las fracturas topografiadas en cuello de fémur correspondieron a $81,52 \%$ y las ocurridas en región trocantérica a $18,48 \%$. La localización mesocervical fue la más frecuente en las fracturas de cuello femoral. Se realizó artroplastia con implante protésico a todas las fracturas de cuello $(81,52 \%)$ y osteosíntesis a todas las fracturas de la región trocantérica $(18,48 \%)$ El tiempo de resolución de la fractura no varió significativamente entre am- 
Tabla 2. Comorbilidades asociadas.

\begin{tabular}{lc}
\hline Comorbilidades & Frecuencia \\
\hline Hipertensión arterial & $69,03 \%$ \\
Enfermedad cardiovascular & $23,37 \%$ \\
Neurológica & $20,65 \%$ \\
Diabetes mellitus 1 y 2 & $20,11 \%$ \\
Hipotiroidismo & $13,59 \%$ \\
Enfermedad psiquiátrica y/o consumo de sedantes & $9,24 \%$ \\
Enfermedad renal crónica & $9,24 \%$ \\
Respiratoria & $7,07 \%$ \\
Otras comorbilidades & $18,48 \%$ \\
\hline
\end{tabular}

bos procedimientos $(\mathrm{p}=0,775)$, con una mediana de 9 días, rango intercuartílico (RI) entre 4 a 19 para artroplastias y de 11 días (RI 6 a 13 días) para las osteosíntesis (figura 2).

Se obtuvo información mediante encuesta a un familiar en 121 casos $(65,76 \%)$ y al propio paciente en 63 casos $(34,24 \%)$. La caída fue la causa precipitante de la fractura en $94,57 \%$, la mayoría ocurrió dentro del domicilio $(70,65 \%)$ y durante el día $(76,65 \%)$. El diagnóstico de OP solamente lo conocía el $15,22 \%$ de la población (figura 3). No tenían prescripción de tratamiento el $69,88 \%$ (figura 4 ). Treinta y nueve pacientes $(23,49 \%)$ tenían indicado suplementos de calcio solo o combinado. Treinta y cuatro $(20,48 \%)$ incluían en su tratamiento vitamina D. Ocho pacientes $(4,82 \%)$ tenían prescriptos bifosfonatos (figura 5). La combinación de fármacos antiosteoporóticos empleados se puede apreciar en la tabla 3. Solo cinco pacientes presentaban un tratamiento completo, entendiendo por tal la asociación de algún antirresortivo, calcio y vitamina D.

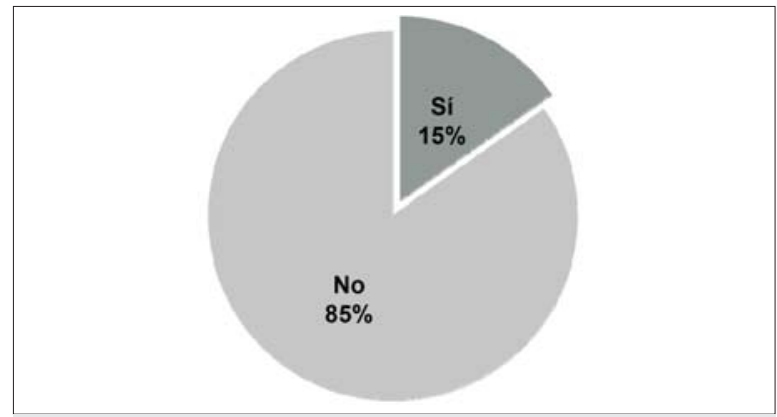

Figura 3. Conocimiento del diagnóstico de osteoporosis luego de la fractura.

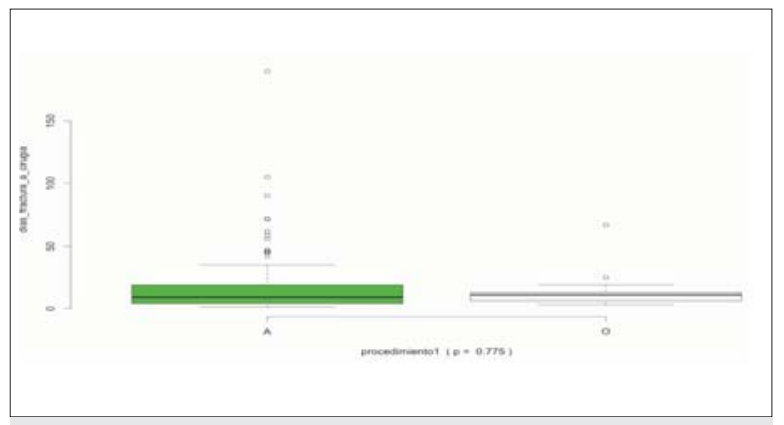

Figura 2. Tiempo de resolución de la fractura. A: artroplastia. O: osteosíntesis.

El antecedente de fractura previa por fragilidad estuvo presente en 48 pacientes $(26,09 \%)$ y ocho pacientes presentaban más de una fractura anterior. La topografía más frecuente fue en la cadera contralateral y en segundo lugar en el puño. Nueve pacientes $(4,89 \%)$ presentaron nuevas fracturas, cuatro de ellos segunda fractura de cadera (tabla 4).

Con respecto a la evolución posterior al evento fracturario, el 77,72\% de los pacientes logró retomar algún grado de deambulación una vez resuelta la fractura, el $66,43 \%$ requirió apoyo instrumental. Diecisiete pacientes $(9,24 \%)$ fueron institucionalizados en residenciales para la tercera edad, luego del alta definitiva (tabla 5). La mortalidad en el período de seguimiento analizado fue de $15,22 \%$, con $11,0 \%$ en el primer año (figura 6 ).

\section{Discusión}

$\mathrm{Al}$ igual que lo ya ampliamente demostrado en la literatura $^{(11,12)}$, este trabajo evidenció mayor prevalencia de fractura de cadera en la mujer, con una mediana de edad para ambos sexos de 82 años. No encontramos diferencias significativas en la edad comparada por sexos, contrariamente a lo reportado, donde el sexo femenino presenta tres a seis años más que el hombre al momento de la fractura ${ }^{(13,14)}$.

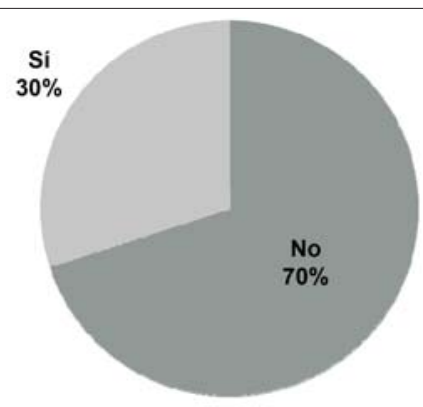

Figura 4. Prescripción de algún fármaco antiosteoporótico luego de la fractura. 


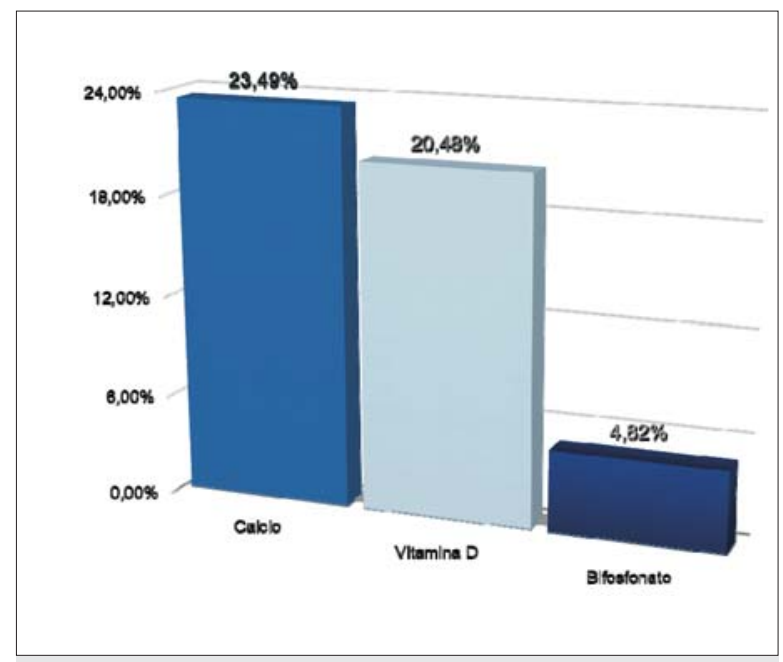

Figura 5. Tratamiento farmacológico solo o combinado.

Tabla 4. Fracturas previas y posteriores a la fractura de cadera.

\begin{tabular}{lcc}
\hline Fracturas previas & 48 pacientes & $\begin{array}{c}26,09 \% \\
(\text { IC } 19,09-33,06)\end{array}$ \\
\hline Topografía: & 16 & \\
Fémur contralateral & 13 & $33,33 \%$ \\
Puño & 5 & $27,08 \%$ \\
Húmero & 2 & $10,42 \%$ \\
Fémur homolateral & 1 & $4,17 \%$ \\
Vértebra & 1 & $2,08 \%$ \\
No sabe & 10 & $2,08 \%$ \\
Otras & 9 pacientes & $4,89($ IC $2,26-9,08)$ \\
\hline Fracturas posteriores & & \\
\hline Topografía: & 4 & $2,83 \%$ \\
Fémur proximal & & \\
\hline
\end{tabular}

En cuanto a las comorbilidades asociadas, la HTA fue la más frecuente, seguida por la ECV, al igual que lo referido en el trabajo de Fukui ${ }^{(15)}$. Si bien esto podría deberse a la alta prevalencia de estas patologías, varios estudios ${ }^{(16,17)}$ encontraron una relación entre la ECV y la fractura de cadera, estableciéndose un enlace fisiopatológico y genético común entre $\mathrm{ECV}$ y $\mathrm{OP}^{(18)}$.

Respecto al tipo de fractura, predominaron las localizadas en cuello femoral frente a las trocantéricas; esta diferencia es debida al sesgo poblacional de donde proviene la muestra.
Tabla 3. Combinación del uso de fármacos antiosteoporóticos.

\begin{tabular}{lc}
\hline Fármaco & Frecuencia relativa \\
\hline Calcio y vitamina D & $11,45 \%$ \\
Calcio y bifosfonatos & $0,60 \%(1)$ \\
Calcio, vitamina D y bifosfonatos & $3,01 \%(5)$ \\
Calcio solo & $8,43 \%$ \\
Vitamina D y bifosfonatos & $0,60 \%(1)$ \\
Vitamina D solo & $5,42 \%(9)$ \\
Bifosfonatos solo & $0,60 \%(1)$ \\
\hline
\end{tabular}

Tabla 5. Evolución del paciente posterior a la fractura.

\begin{tabular}{lc}
\hline Retoma deambulación & $77,72 \%$ \\
Uso de apoyo instrumental & $66,43 \%$ \\
Institucionalización & $9,24 \%$ \\
Mortalidad al año & $11 \%$ \\
\hline
\end{tabular}

Un trabajo nacional ${ }^{(19)}$ realizado por el FNR en el año 2002 comparte resultados similares en algunas variables analizadas: predominio de sexo femenino con similar rango etario al momento de la fractura, número de comorbilidades asociadas, siendo la HTA la más frecuente, recuperación de la marcha en un alto porcentaje de pacientes y retraso en la resolución quirúrgica mediante artroplastia con implante protésico con similar mediana. Nuestro trabajo evaluó el tiempo de demora en la resolución quirúrgica mediante artroplastia u osteosíntesis, no encontrando diferencias significativas entre ambos procedimientos, con una alta demora en ambos.

Está fuertemente demostrado que la cirugía precoz (primeras 24 horas) mejora los resultados en cuanto a disminución de la mortalidad, mayor recuperación funcional y menor incidencia de complicaciones ${ }^{(20)}$.

La caída como causa precipitante de la fractura estuvo presente en el $95 \%$ de nuestra población, lo que muestra su relevancia como factor causal. Este hecho concuerda con otros estudios en la literatura ${ }^{(21)}$. En cuanto al lugar y el horario de la caída, nuestros resultados revelaron que la mayoría ocurrió en el domicilio (71\%) y durante el día (77\%), resultados semejantes se constataron en el estudio Medos ${ }^{(22)}$ realizado en 14 países de Europa. 
Respecto al conocimiento del diagnóstico de OP, solo $15 \%$ de los pacientes consideraban que padecían esta enfermedad luego de la fractura. Giangregrio ${ }^{(23)}$ y Chevalley $^{(24)}$ evaluaron este mismo tópico a través de un cuestionario escrito en el primer caso y telefónico en el segundo, encontrando similares resultados $(17 \%$ de conocimiento de la enfermedad en ambos trabajos).

Si bien es conocido que la primera fractura por fragilidad aumenta considerablemente el riesgo de una nueva fractura, la mayoría de los pacientes no reciben tratamiento médico antiosteoporótico luego del primer evento ${ }^{(25-28)}$

Nuestro trabajo reporta solamente $30 \%$ de individuos que reciben fármacos para la $\mathrm{OP}$. Un estudio realizado en España, con más de 2.500 pacientes con fractura de cadera, mostró que solo $38 \%$ presentaba algún tipo de tratamiento osteoporótico ${ }^{(29)}$. Una revisión sistemática de la literatura ${ }^{(26)}$ evaluó el porcentaje de pacientes con tratamiento luego de una fractura de cadera con resultados variables, que van desde $6 \%$ a $61,7 \%$, según el diseño del trabajo, y demostró que el tratamiento con calcio, solo o en combinación con vitamina $\mathrm{D}$, era la terapia más prescripta, al igual que lo hallado en nuestro estudio. Solo cinco pacientes de la muestra recibían el tratamiento pautado en las distintas guías internacionales de OP que incluyen adecuado aporte de calcio, vitamina $\mathrm{D}$ y antirresortivos en forma simultánea ${ }^{(2,27)}$. En igual sentido, Jennings y colaboradores ${ }^{(28)}$ informaron sobre un total de 51.346 pacientes, $2 \%$ de tratamiento completo.

La cuarta parte de nuestros pacientes presentaban fracturas previas, la más frecuente fue la de cadera contralateral, seguida de la fractura de puño, resultados semejantes fueron obtenidos en el trabajo de Follin y colaboradores $^{(14)}$. Reportes con mayores porcentajes de fracturas previas, cercanos a $50 \%$, fueron referidos en otros estudios ${ }^{(5)}$.

Nueve pacientes presentaron nuevas fracturas en la evolución, cuatro de ellos una segunda fractura de cadera, estos números son pequeños y no nos permiten obtener conclusiones significativas ni comparar con otros estudios. Un metaanálisis ${ }^{(31)}$ mostró variabilidad en los porcentajes de segunda fractura de cadera, que van desde $2,3 \%$ a $13,8 \%$, dependiendo del tiempo de seguimiento del trabajo. Porcentajes también menores fueron reportados en los estudios de Scaglioni ${ }^{(32)}$ y Ravenda $^{(7)}$.

En lo relacionado con la evolución posterior a la fractura, $9 \%$ de los pacientes fueron institucionalizados, estos porcentajes son menores a lo referido en la literatu$\mathrm{ra}^{(33)}$. En cuanto a la capacidad de marcha luego de resuelta la fractura, este estudio mostró que $77 \%$ de los pacientes pudo volver a caminar, en su mayoría con apoyo instrumental. Si bien no hemos valorado en este trabajo el grado de deambulación, el porcentaje de recuperación

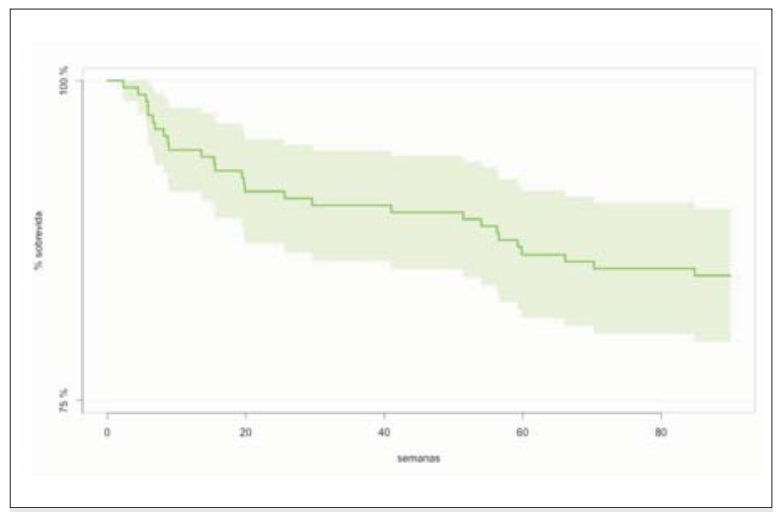

Figura 6. Sobrevida durante el período de seguimiento

funcional es similar a lo reportado en una revisión de la literatura ${ }^{(34)}$ a dos años de seguimiento (72\%).

$\mathrm{Al}$ analizar la mortalidad posterior a la fractura encontramos que la misma fue de $15 \%$ en todo el período de seguimiento, siendo esta máxima en el primer año $(11 \%)$. Similares porcentajes fueron encontrados en trabajos internacionales: Farahmand y colaboradores ${ }^{(35)}$ $(10,6 \%)$ y Richmond y colaboradores ${ }^{(36)}(11,5 \%)$.

\section{Limitaciones del trabajo}

Este estudio tiene varias limitaciones, por lo que los resultados deben interpretarse con cautela. En primer lugar, la existencia de un sesgo de selección en el trabajo vinculado al centro de donde proviene la muestra. En segundo lugar, los datos respecto a comorbilidades asociadas fueron recabados de las historias clínicas del Banco de Prótesis y no de las del centro de origen del paciente, lo que puede determinar información incompleta de los antecedentes. En tercer lugar, respecto a prescripción de tratamiento, no consignamos dosis recibida de vitamina D ni de calcio, ni valoramos el consumo de este último con la dieta. Así mismo no se describe el tipo de bifosfonato que recibía el paciente. Si bien estos datos son importantes para determinar un tratamiento adecuado, no alteran el resultado de pacientes que estaban bajo tratamiento médico, que era lo que queríamos investigar. Por último, el hecho de obtener información mediante encuesta telefónica puede arrojar datos no completamente confiables.

\section{Conclusiones}

La fractura de cadera ocurre principalmente en la mujer, en la octava década de la vida.

El tratamiento está centrado en el evento agudo y en la intervención operatoria. Existe una demora quirúrgica en la resolución que dista mucho de lo recomendado a nivel internacional. Nuestros pacientes desconocen que 
padecen OP como determinante de la fractura y no reciben el tratamiento adecuado para prevenir nuevos episodios. La caída es la precipitante de la fractura en casi la totalidad de los casos y ocurre fundamentalmente de día y en el domicilio. La mayoría de los pacientes logra recuperación funcional y puede retomar la deambulación, aunque con apoyo instrumental. La mortalidad es máxima en el primer año posterior al evento.

Este trabajo pone en evidencia la subestimación del diagnóstico de OP, esperamos que el mismo invite a la reflexión del equipo de salud cuando se enfrenta a un paciente con fractura de cadera. Es necesario acortar los tiempos de resolución quirúrgica y establecer diagnósticos y tratamientos adecuados para poder actuar en la prevención de nuevos eventos. Deseamos que Uruguay, en el futuro, al igual que otros países, cuente con sistemas de coordinación o enlace del paciente con fractura por fragilidad que brinden una atención integrada y completa y reditúe en la reducción de los costos en salud.

\section{Abstract}

Introduction: hip fracture due to frailty is considered a marker of osteoporotic disease in the elderly population. It determines a high morbidity and mortality and high health costs.

Objectives: to analyze characteristics and evolution after a hip fracture due to fragility in patients over 65 years of age assisted in the Banco de Protesis during the January-June 2013 period.

Methods: historical cohort follow-up study, consisting of 184 patients. The data was obtained from the medical records and the follow-up was done by telephone. The maximum follow-up time was 84 weeks.

Results: $82 \%$ of patients were female, with median age of 80.7 years for men and 82.7 for women. The most frequent comorbidities were hypertension and cardiovascular disease. There were no differences in the right or left location, the majority corresponded to femoral neck fractures. The median resolution time was 9 days for arthroplasty and 11 days for osteosynthesis. The fall was the determinant in $94.5 \%$ of the cases. Twenty-six percent of the patients had previous fragility fractures. $85 \%$ of individuals did not know about osteoporosis (OP) and $69 \%$ did not receive treatment for this disease. $9 \%$ was institutionalized, $77 \%$ managed to resume walking. $15 \%$ died in the follow-up period.

Conclusions: osteoporosis is not recognized or treated in patients with fragility fracture hip.

\section{Resumo}

Introdução: a fratura de quadril por fragilidade é considerada um marcador de enfermidade osteoporótica em idosos. Determina uma elevada morbimortalidade e altos custos em saúde.

Objetivos: analisar as características e a evolução posterior a uma fratura de quadril por fragilidade em pacientes $\geq 65$ anos atendidos no Banco de Prótesis durante o período janeiro-junho 2013.

Métodos: estudo de seguimento de coorte histórica com 184 pacientes. Os dados foram obtidos dos prontuários de pacientes e o seguimento foi feito por via telefônica. O tempo máximo de seguimento foi de 84 semanas.

Resultados: $82.6 \%$ dos pacientes era do sexo feminino; a mediana de idade foi de 80.7 anos para os homens e 82.7 para as mulheres. As comorbidades mais frequentes foram hipertensão arterial e patologia cardiovascular. Não foram observadas diferenças na localização da fratura - direita ou esquerda sendo a maioria fraturas do colo do fêmur. A mediana do tempo de resolução foi de 9 dias para autoplastia e 11 dias para osteossíntese. A queda foi o fator determinante em $94.5 \%$ dos casos. Vinte e seis por cento dos pacientes tinham fraturas previas por fragilidade. $85 \%$ dos indivíduos não sabia que era portador de osteoporose (OP) e $69 \%$ não recebia tratamento para esta enfermidade. $9 \%$ foi institucionalizado, 77\% retomou a deambulação e 15\% faleceu durante o seguimento.

Conclusões: não se reconhece nem se trata a osteoporose nos pacientes com fratura de quadril por fragilidade.

\section{Bibliografía}

1. Uruguay. Fondo Nacional de Recursos. Informe anual de autorizaciones 2013. Disponible en: http://www.fnr.gub.uy/ sites/default/files/estadisticas/ia2013/ia_aut_2013_1.pdf [Consulta: 16 febrero 2018].

2. NIH Consensus Development Panel on Osteoporosis Prevention, Diagnosis, and Therapy. Osteoporosis prevention, diagnosis and therapy. JAMA 2001; 285(6):785-95.

3. Braithwaite RS, Col NF, Wong JB. Estimating hip fracture morbidity, mortality and costs. J Am Geriatr Soc 2003; 51:364-70.

4. Alarcón Alarcón T, González-Montalvo JI. Fractura osteoporótica de cadera. Factores predictivos de recuperación funcional a corto y largo plazo. An Med Interna (Madrid) 2004; 21:49-58.

5. Port L, Center J, Briffa NK, Nguyen T, Cumming R, Eisman J. Osteoporotic fracture: missed opportunity for intervention. Osteoporosis Int 2003; 14:780-4.

6. Lee YK, Ha YC, Yoon BH, Koo KH. Incidence of second hip fracture and compliant use of bisphosphonate. Osteoporos Int 2013; 24:2099-104.

7. RabendaV, Vanoverloop J, Fabri V, Mertens R, Sumkay F, Vannecke C, et al. Low incidence of anti-osteoporosis 
treatment after hip fracture. J Bone Joint Surg Am 2008; 90:2142-8

8. Elliot-Gibson V, Bogoch ER, Jamal SA, Beaton DE. Practice patterns in the diagnosis and treatment of osteoporosis after a fragility fracture: a systematic review. Osteoporos Int 2004; 15(10):767-78.

9. Lyles KW, Colon-Emeric CS, Magaziner JS, Adachi JD, Pieper CF, Mautalen C, et al. Zoledronic acid and clinical fractures and mortality after hip fracture. N Engl J Med 2007; 357(18):1799-809.

10. Osaki M, Tatsuki K, Hashikawa T, Norimatsu T, Chiba K, Motokawa S, et al. Beneficial effect of risedronato for preventing recurrent hip fracture in the elderly Japanese women. Osteoporos Int 2012; 23:695-703.

11. Melton LJ 3 ${ }^{\text {rd }}$. Epidemiology worldwide. Endocrinol Metab Clin North Am 2003; 32:1-13.

12. Johnell O, Kanis JA. An estimate of the worldwide prevalence and disability associated with osteoporotic fractures. Osteoporos Int 2006; 17:1726-33.

13. Lofman O, Berglund K, Larsson L, Toss G. Changes in hip fracture epidemiology: redistribution between ages, genders and fracture types. Osteoporos Int 2002; 13:18-25.

14. Follin SL, Black JN, McDermott MT. Lack of diagnosis and treatment of osteoporosis in men and women after hip fracture. Pharmacotherapy $2003 ; 23: 190-8$

15. Fukui N, Watanabe Y, Nakano T, Sawaguchi T, Matsuchita T. Predictors for ambulatory ability and the change in ADL after hip fracture in patient with different levels of mobility before injury: a 1-yer prospective cohort study. J Orthop Trauma 2012; 26:163-71.

16. Sennerby U, Melhus H, Gedeborg R, Byberg L, Garmo H, Ahlbom A, et al. Cardiovascular diseases and risk of hip fracture. JAMA 2009; 302(15):1666-73.

17. Xu B, Han L, Liu H, Wang J, Bao XY, Xi HX, et al. Cardiovascular disease and hip fracture among older inpatients in Beijing, China. Biomed Res Int 2013; 2013. doi:10.1155/ 2013/493696.

18. Sprini D, Rini GB, Di Stefano L, Cianferotti L, Napoli N. Correlation between osteoporosis and cardiovascular disease. Clin Cases Miner Bone Metab 2014; 11(2):117-9.

19. Albornoz H, Gambogi R, Debenedetti A, Gonzalez MC, Scarpitta C. Seguimiento prospectivo de artroplastia de cadera por fractura (2002). En: Fondo Nacional de Recursos. La artroplastia de cadera y rodilla. Montevideo: FNR, 2009:39-92. (Publicación técnica, 9). Disponible en: www.fnr.gub.uy/sites/default/files/publicaciones/FNR_publicacion_tecnica_9.pdf [Consulta: 24 setiembre 2018].
20. Simunovic N, Devereaux PJ, Sprague S, Guyatt GH, Schemitsch E, Debeer J, et al. Effect of early surgery after hip fracture on mortality and complications: systematic review and meta-analysis. CMAJ 2010; 182:1609-16.

21. Norton R, Campbell A, Lee-Joe T, Robinson E, Butler M. Circumstances of falls resulting in hip fractures among older people. J Am Geriatr Soc 1997; 45(9):1108-12.

22. Allander E, Gullberg B, Johnell O, Kanis J, Ranstam J, Elffors L. Circumstances around the fall in a multinational hip fracture risk study: a diverse pattern for prevention. Accid Anal Prev 1998; 30(5):607-16.

23. Giangregorio L, Papaioannou A, Thabane L, DeBeer J, Cranney A, Dolovich L, et al. Do patients perceive a link between a fragility fracture and osteoporosis? BMC Musculoskelet Disord 2008; 9:38

24. Chevalley T, Hoffmeyer P, Bonjour JP, Rizzoli R. An osteoporosis clinical pathway for the medical management of patients with low-trauma fracture. Osteoporos Int 2002; $13: 450-5$.

25. Harrington JT, Broy SB, Derosa AM, Licata AA, Shewmon DA. Hip fracture patients are not treated for osteoporosis: a call to action. Arthritis Rheum 2002; 47:651-4.

26. Elliot-Gibson V, Bogoch ER, Jamal SA, Beaton DE. Practice patterns in the diagnosis and treatment of osteoporosis after a fragility fracture: a systematic review. Osteoporosis Int 2004; 15:767-78.

27. Lems WF, Dreinhofer KE, Bischoff-Ferrari H, Blauth M, Czerwinski E, da Silva J, et al. EULAR/EFORT recommendations for management of patients older than 50 years with a fragility fracture and prevention of subsequent fractures. Ann Rheum 2017; 76(5):802-10.

28. Jennings LA, Auerbach AD, Maselli J, Pekow PS, Lindenauer PK, Lee SJ. Missed opportunities for osteoporosis treatment in patients hospitalized for hip fracture. J Am Geriatr Soc 2010; 58(4):650-7.

29. Khosla S, Shane E. A crisis in the treatment of osteoporosis. J Bone Miner Res 2016; 31:1485-7.

30. León Vázquez F, Bonis J, Bryant Cerezo V, Herrero Hernández S, Jamart Sánchez L, Díaz Holgado A. Prevención de fractura osteoporótica en España: uso de fármacos antes y después de una fractura de cadera. Rev Osteoporos Metab Miner 2015; 7(2):54-62.

31. Zhu Y, Chen W, Sun T, Zhang Q, Cheng J, Zhang Y. Meta-analysis of risk factors for the second hip fracture (SHF) in elderly patients. Arch Gerontol Geriatr 2014; 59(1):1-6.

32. Scaglione M, Fabbri L, Di Rollo F, Bianchi MG, Dell'omo D, Guido G. The second hip fracture in osteoporotic patients:

\section{Contribución de autores}

Todos los autores participaron en igual medidas en todos los proceso de diseño, recolección de datos, análisis, redacción y revisión

Beatriz Mendoza, https://orcid.org/0000-0002-7898-9336

Marcela Hernández, https://orcid.org/0000-0001-9318-0773

Marianela Maiche, https://orcid.org/0000-0002-8418-0419 
not only an orthopaedic matter. Clin Cases Miner Bone Metab 2013; 10(2):124-8.

33. Cumming RG, Klineberg R, Katelaris A. Cohort study of risk of institutionalisation after hip fracture. Aust N Z Public Health 1996; 20(6):579-82.

34. Alarcón Alarcón T, González-Montalvo JI. Fractura osteoporótica de cadera. Factores predictivos de recuperación fun- cional a corto y largo plazo. An Med Interna (Madrid) 2004; 21:49-58.

35. Farahmand BY, Michae"Isson K, Ahlbom A, Ljunghall S, Baron JA. Survival after hip fracture. Osteoporos Int 2005; 16:1583-90.

36. Richmond J, Aharonoff GB, Zuckerman JD, Koval KJ. Mortality risk after hip fracture. J Orthop Trauma 2003; 17(1):53-6. 